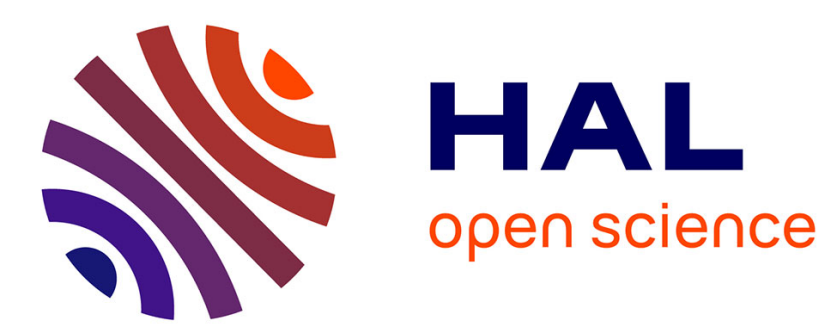

\title{
Perfect spin filtering by symmetry in molecular junctions
}

\author{
Dongzhe Li, Yannick J. Dappe, Alexander Smogunov
}

\section{To cite this version:}

Dongzhe Li, Yannick J. Dappe, Alexander Smogunov. Perfect spin filtering by symmetry in molecular junctions. Physical Review B: Condensed Matter and Materials Physics (1998-2015), 2016, 93, pp.201403. 10.1103/PhysRevB.93.201403 . cea-01485684

\section{HAL Id: cea-01485684 https://hal-cea.archives-ouvertes.fr/cea-01485684}

Submitted on 9 Mar 2017

HAL is a multi-disciplinary open access archive for the deposit and dissemination of scientific research documents, whether they are published or not. The documents may come from teaching and research institutions in France or abroad, or from public or private research centers.
L'archive ouverte pluridisciplinaire HAL, est destinée au dépôt et à la diffusion de documents scientifiques de niveau recherche, publiés ou non, émanant des établissements d'enseignement et de recherche français ou étrangers, des laboratoires publics ou privés. 


\title{
Perfect spin filtering by symmetry in molecular junctions
}

\author{
Dongzhe Li ${ }^{1,2}$ Yannick J. Dappe, ${ }^{1}$ and Alexander Smogunov ${ }^{1, *}$ \\ ${ }^{1}$ Service de Physique de l'Etat Condensé (SPEC), CEA, CNRS, Université Paris-Saclay, CEA Saclay, 91191 Gif-sur-Yvette Cedex, France \\ ${ }^{2}$ Department of Physics, University of Konstanz, 78457 Konstanz, Germany
}

(Received 14 March 2016; revised manuscript received 13 May 2016; published 27 May 2016)

\begin{abstract}
Obtaining highly spin-polarized currents in molecular junctions is crucial and important for nanoscale spintronics devices. Motivated by our recent symmetry-based theoretical argument for complete blocking of one spin conductance channel in model molecular junctions [A. Smogunov and Y. J. Dappe, Nano Lett. 15, 3552 (2015)], we explore the generality of the proposed mechanism and the degree of achieved spin-polarized current for realistic molecular junctions made of various ferromagnetic electrodes ( $\mathrm{Ni}, \mathrm{Co}, \mathrm{Fe})$ connected by different molecules (quaterthiophene or $p$-quaterphenyl). A simple analysis of the spin-resolved local density of states of a free electrode allowed us to identify the $\mathrm{Fe}(110)$ as the most optimal electrode, providing perfect spin filtering and high conductance at the same time. These results are confirmed by ab initio quantum transport calculations and are similar to those reported previously for model junctions. It is found, moreover, that the distortion of the p-quaterphenyl molecule plays an important role, reducing significantly the overall conductance.
\end{abstract}

DOI: 10.1103/PhysRevB.93.201403

Molecular spintronics is a very promising and emerging field, whose aim is to manipulate the spin degrees of freedom in molecular-based devices [1,2]. Such devices should possess a large spin-relaxation length, which is important for using spin degrees of freedom in transport properties. In particular, due to low spin-orbit coupling in organic molecules, the electron spin-relaxation length will be rather large, making such organic-based devices very promising for spintronics applications. The property of high interest in spintronics is the spin filtering or the spin polarization of (zero bias) electric current, $\mathrm{P}=\left(G_{\uparrow}-G_{\downarrow}\right) /\left(G_{\uparrow}+G_{\downarrow}\right)$, where $G_{\uparrow}$ and $G_{\downarrow}$ are the conductances of the majority and minority spin channels, respectively. Another important property is the magnetoresistance (MR) - the strong change in electrical conductance $G$ between parallel and antiparallel magnetic alignments of two ferromagnetic electrodes- $\mathrm{MR}=\left(G_{\mathrm{P}}-G_{\mathrm{AP}}\right) / G_{\mathrm{AP}}$. In this context, achieving as large as possible $\mathrm{P}$ (ideally, 100\%) and MR (ideally, infinite) represents a crucial issue.

An unexpected large MR of up to $300 \%$ and large spin-dependent transport lengths have been reported in spin valves using $\mathrm{Alq}_{3}[3,4]$. The MR of about $60 \%, 67 \%$, and $80 \%$ have been reported for $\mathrm{Co}\left|\mathrm{C}_{60}\right| \mathrm{Co}[5], \mathrm{Fe}\left|\mathrm{C}_{70}\right| \mathrm{Fe}[6]$, and $\mathrm{Ni}\left|\mathrm{C}_{60}\right| \mathrm{Ni}$ [7] magnetic junctions, respectively. At the single molecular scale, due to strong hybridization between molecular orbitals and ferromagnetic electrode states, it has been found that the giant MR (GMR) can reach rather large values, ranging from $50 \%$ to $80 \%$ [8-10], in the contact regime. In the tunneling regime, a tunneling MR (TMR) value of up to $100 \%$ has been found in the case of a single $\mathrm{C}_{60}$ molecule deposited on the chromium surface [11]. Moreover, a selective and efficient spin injection at the ferromagneticorganic interface has also been reported by locally controlling the inversion of the spin polarization close to the Fermi level [12]. This effect, however, was found to depend strongly on the details of the nanocontact configuration as well as on the molecule-electrode coupling.

\footnotetext{
*alexander.smogunov@cea.fr
}

Quite generally, the conductance in ferromagnetic nanocontacts is dominated by weakly polarized $s$ orbitals resulting in a partial spin-polarized current [13-15]. Therefore, blocking transport via $s$ orbitals and promoting transport via $d$ (or $p$ ) orbitals seems to be a good strategy to obtain high spin polarizations. Very recently, $100 \%$ spin-polarized currents have been reported experimentally in the nickel oxide atomic junctions formed between two nickel electrodes [16] by the break-junction setup. The current work is motivated by our recent study of spin-polarized electron transport through a special class of $\pi$-conjugated molecules bridging two Ni electrodes [17]. Due to a symmetry mismatch between molecular orbitals and conductance channels of the $\mathrm{Ni}$ electrodes, the electron transport was carried by only $d$ orbitals while the $s$-conductance channels were fully blocked at the electrodemolecule junction. As a consequence, a perfect $100 \%$ spin polarization $\left(G_{\downarrow} \approx 0.65 G_{0}, G_{\uparrow}=0\right)$ and an infinite MR have been found for ideal $\mathrm{Ni}$ electrodes (represented by semi-infinite $\mathrm{Ni}$ chains), while rather moderate values $\left(G_{\downarrow} \approx 0.19 G_{0}, G_{\uparrow} \approx\right.$ $0.02 G_{0}$ ) were found for more realistic $\mathrm{Ni}(111)$ electrodes.

The purpose of this Rapid Communication is to elaborate on this weak point and to suggest realistic molecular junctions (electrode and molecule) employing in full our symmetry argument and approaching as close as possible to the ideal result found previously for model electrodes. We argue that the key property to look at is the spin-polarized density of states (DOS) of appropriate symmetry at the apex atom of the free electrode - the property which is readily accessible from standard electronic structure density functional theory (DFT) calculations. It will be shown that the $\mathrm{Fe}(110)$ electrode is the optimal one, providing the highest spin polarization of incoming channels at the Fermi energy, while polythiophene molecules are probably the best ones due to their highest occupied molecular orbital (HOMO) placed very closely to the Fermi level. In such junctions a perfect spin filtering with a rather big spin-down conductance is predicted, very close to the ideal result found earlier for idealized nanowire $\mathrm{Ni}$ electrodes. Moreover, we demonstrate that our symmetry argument has a quite general character and applies in fact to a variety of ferromagnetic electrodes (such as $\mathrm{Ni}, \mathrm{Co}, \mathrm{Fe}$, etc.) 
(a)

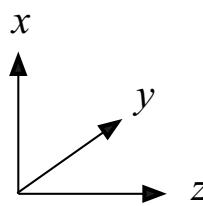

(b)

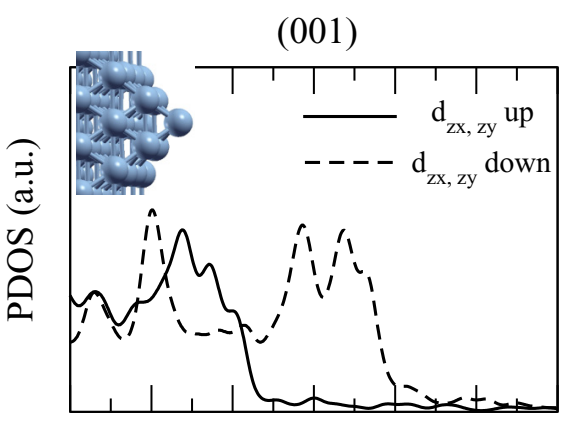

(111)

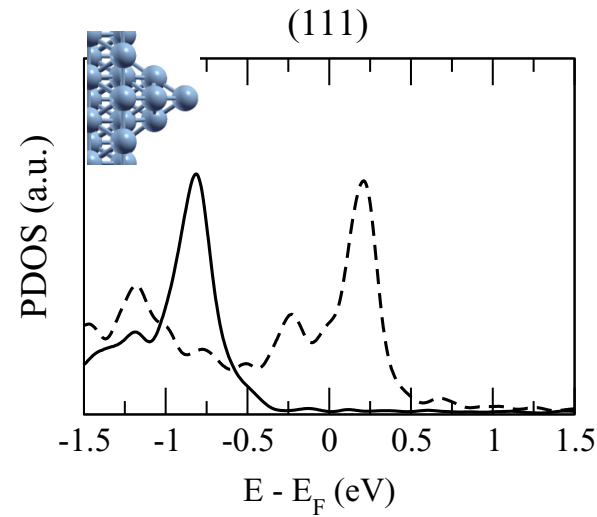

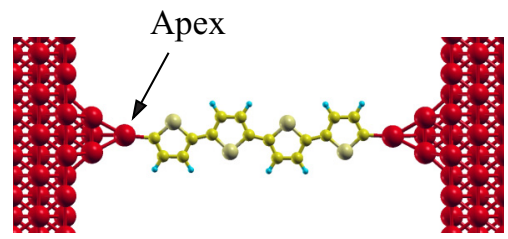

(c)

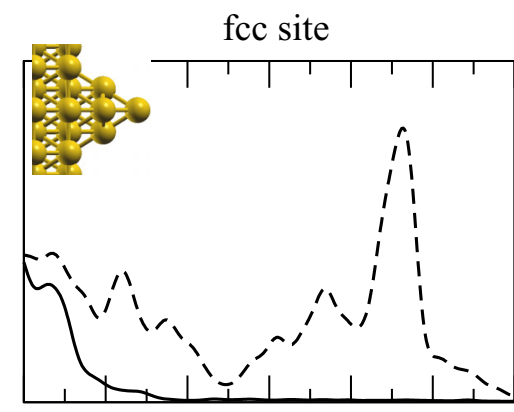

hcp site

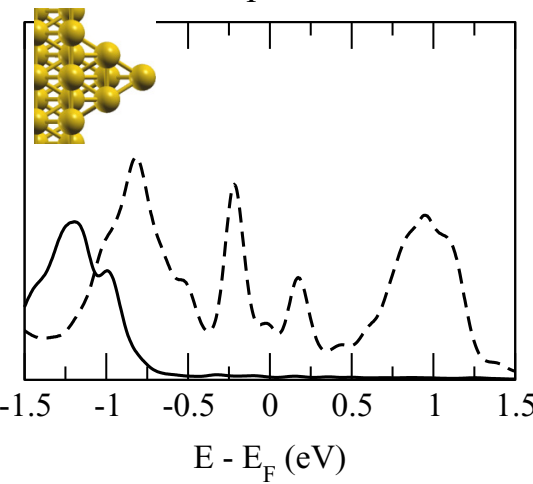

(d)

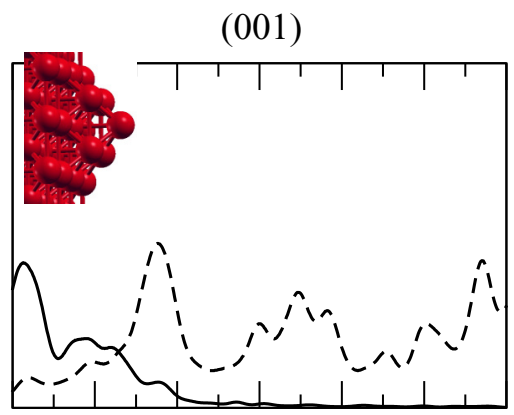

(110)

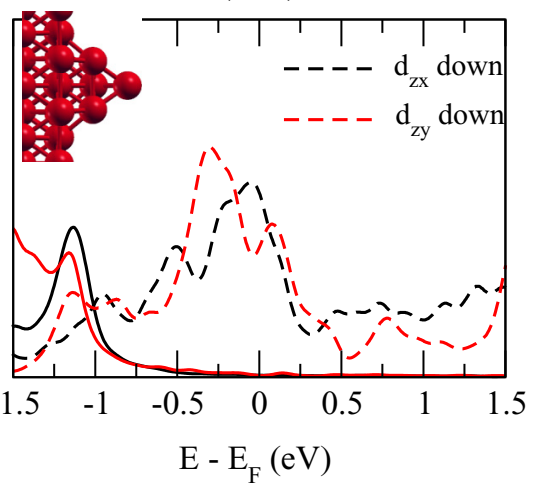

FIG. 1. (a) Model geometry of the ferromagnetic|molecule|ferromagnetic nanojunction used. Spin-polarized projected density of states (PDOS) on $d_{z x, z y}$ orbitals of one of (equivalent) apex atoms of the electrodes for (b) fcc Ni, of (001) (top) and (111) (bottom) orientations; (c) hep Co, of fcc (top) and hcp (bottom) positions of the pyramidlike tip; and (d) bcc Fe, of (001) (top) and (110) (bottom) orientations. The insets show the atomic structures of the corresponding electrodes which are terminated with only one apex atom. The pyramids contain either four [ $\mathrm{Ni}(111)$, hcp $\mathrm{Co}$, and $\mathrm{Fe}(110)]$ or five [ $\mathrm{Ni}(001)$ and $\mathrm{Fe}(001)]$ atoms. The $d_{z x}$ and $d_{z y}$ orbitals are degenerate for all presented electrodes, except for $\mathrm{Fe}(110)$, due to a symmetry reason. Spin-up (spin-down) results are plotted by solid (dashed) lines.

with similar spin-asymmetric band structures at the Fermi level and to various $\pi$-conjugated molecules.

DFT-based spin-dependent quantum transport. The spinpolarized $a b$ initio calculations were carried out using the plane wave electronic structure package QUANTUM ESPRESSO [18] in the framework of the density functional theory (DFT). We used the local density approximation (LDA) with Perdew-Zunger parametrization [19] of the exchange-correlation functional with ultrasoft pseudopotentials (USPPs) to describe the electron-ion interaction. The molecular junctions were simulated by a supercell, such as the one shown in Fig. 1(a). Details concerning the calculations can be found in Ref. [20]. For calculations of ballistic transport across the junction, the unit cell of Fig. 1(a) was considered as a scattering region joint perfectly on both sides to semi-infinite electrodes. Electron transmission was then evaluated using the scattering state approach as implemented in the PWCOND code [21]. The ballistic conductance (at the infinitesimal voltage) is given by the Landauer-Büttiker formula $G=G_{0}\left[T_{\uparrow}\left(E_{\mathrm{F}}\right)+T_{\downarrow}\left(E_{\mathrm{F}}\right)\right]$, where $G_{0}=e^{2} / h$ is the quantum of conductance. A $(8 \times 8) k$-point mesh in the $x y$ plane was found to be enough to obtain well converged transmission functions. Notice that the DFT scheme can overestimate the transmission due to the underestimation of the band gap [22,23]. However, such effects may affect quantitatively the electron transport properties in our molecular junction, but the main physical trends will remain unaffected due to our robust symmetry argument. Moreover, as we will see later, the conductance is provided by the HOMO molecular orbital whose position with respect to the Fermi level we believe is described quite well by our DFT calculations.

The key idea of the symmetry argument is to block the electrode $s$ channels (both of the majority and minority spins) at the Fermi energy by using $\pi$-conjugated molecules having only $\pi$ states available around the $E_{\mathrm{F}}$. The remaining four transport $d$ channels are all of the minority spin (the majority spin $d$ states are all occupied and lie well below 
the $\left.E_{\mathrm{F}}\right)$ which would result in a fully spin-polarized current across such molecular junctions. In this context, the spinpolarized density of states at the electrode apex atom plays a crucial role, providing the information on the amount and the spin polarization of states available for transport. We start therefore by analyzing the projected density of states (PDOS) at the electrode apex atom for free electrodes of different ferromagnetic metals in order to select the best candidate as a spin injection material.

Electronic structure of ferromagnetic electrodes. The ferromagnetic electrodes are modeled by five atomic layers containing 16 atoms in each atomic plane and terminated by a pyramidlike tip. Note that the pyramids contain four or five atoms for cubic or hexagonal electrodes, respectively (see the insets in Fig. 1). The two bottom layers were fixed while the other three layers and the pyramid were relaxed until the atomic forces are less than $1 \mathrm{meV} / \AA$.

We emphasize that it is enough to look at only the $d_{z x, z y}$ states of the apex atom (Fig. 1) since the other apex $d$ states have no overlap with the out-of-plane molecular $\pi$ states around the Fermi energy. Note that the molecule is assumed to be aligned with the transport direction $Z$ but otherwise can rotate around it. In the case where it lies exactly in the $Y Z$ plane, for example, the molecular states will hybridize with only $d_{z x}$ states, which are of appropriate symmetry (odd with respect to the molecule $Y Z$ plane). We notice also that, by symmetry, the $d_{z x}$ and $d_{z y}$ orbitals are degenerated for all the presented electrodes, except for the $\mathrm{Fe}(110)$ electrode.

As expected, a quite general feature has been found for all the ferromagnetic electrodes - the apex DOS around the Fermi energy is dominated by partially filled minority $d$ states. For $\mathrm{Ni}(001)$ electrodes [Fig. 1(b), upper panel], the spin-down PDOS peak lies in the vicinity of the Fermi energy while the spin-up PDOS is very small but not negligible and starts growing significantly at only $E-E_{\mathrm{F}}<-0.45 \mathrm{eV}$. In the case of $\mathrm{Ni}(111)$ [Fig. 1(b), lower panel], a relatively smaller [compared to the $\mathrm{Ni}(001)$ ] spin-down PDOS is found at the Fermi energy with the maximum around $0.25 \mathrm{eV}$ above the Fermi energy.

For hcp Co electrodes as shown in Fig. 1(c), two different adsorption sites, namely, fcc and hcp, for the pyramid were considered. We found that the hcp site is slightly more favorable with respect to the fcc one, with an energy gain of about $17.84 \mathrm{meV}$. Compared to the fcc Ni electrode, the apex PDOS for spin-down polarization has a relatively smaller amplitude at the Fermi energy while the spin-up states are almost absent.

For the case of a bcc Fe electrode we have considered two crystallographic orientations, (001) and (110), shown in Fig. 1(d) on the upper and lower panels, respectively. The apex atom PDOS of the $\mathrm{Fe}(001)$ electrode exhibits a similar spin polarization as for $\mathrm{Ni}(111)$, even though the peaks are rather different. On the other hand, almost perfect behavior of the PDOS has been found for the $\mathrm{Fe}(110)$ orientation, namely, a rather smooth and large PDOS for spin-down states in a quite large energy window $[-0.75 \mathrm{eV},+0.5 \mathrm{eV}]$ with a maximum around the Fermi energy and almost zero PDOS around the Fermi energy for spin-up states. In addition, the non-negligible PDOS for the spin-up states start about $-0.8 \mathrm{eV}$ with respect to the Fermi energy.
We conclude, therefore, that among all the electrodes considered, the $\mathrm{Fe}(110)$ looks to be the optimal one for providing a highly spin-polarized incoming current. It is expected also to improve considerably over the $\mathrm{Ni}(111)$ electrode considered in our previous work [17].

$\mathrm{Fe}(110) \mid$ quaterthiophene $\mid \mathrm{Fe}(110)$. After having selected the best electrode, which in our case turns out to be the Fe(110), we will now confirm our findings by calculating explicitly the spin-polarized transmission functions. We first consider the quaterthiophene molecule which contains four cycles of thiophene (standing perpendicular to the surface), suspended between two Fe(110) electrodes, as shown in Fig. 1(a) as a geometric model. Experimentally, two well established techniques, namely, scanning tunneling microscope (STM) and mechanically controllable break junctions (MCBJs), can be used to fabricate and to manipulate the molecular junctions. For instance, recent experiments have shown that such molecular junctions could be well established with a STM tip by picking up one end of the individual polythiophene on the surface and lifting it up [24,25]. In addition, stable and highly conductive molecular junctions were also obtained by the MCBJ technique [26,27]. By symmetry, the $d_{z x}$ orbital of the Fe apex has nonzero overlap with the out-of-plane $\pi$ states of the molecule (if the $Y Z$ plane is chosen to correspond to the molecule plane). The PDOS of the Fe apex, as shown in Fig. 2(a), presents a very similar feature compared to the case

(a)

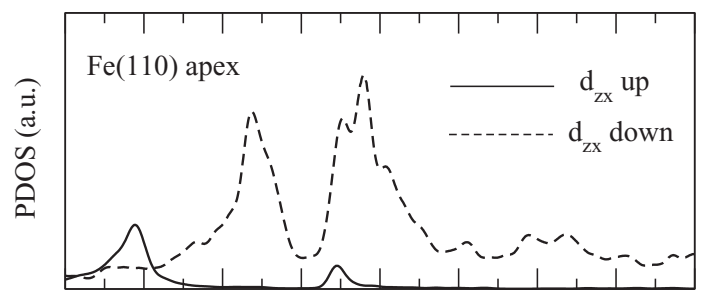

(b)

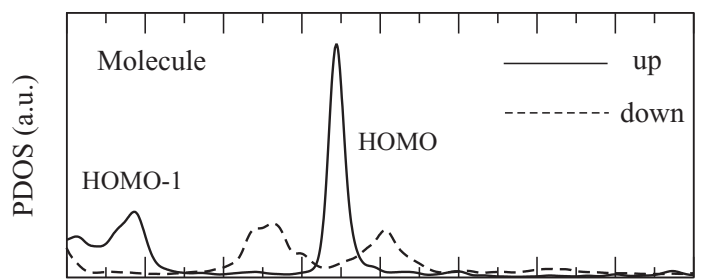

(c)

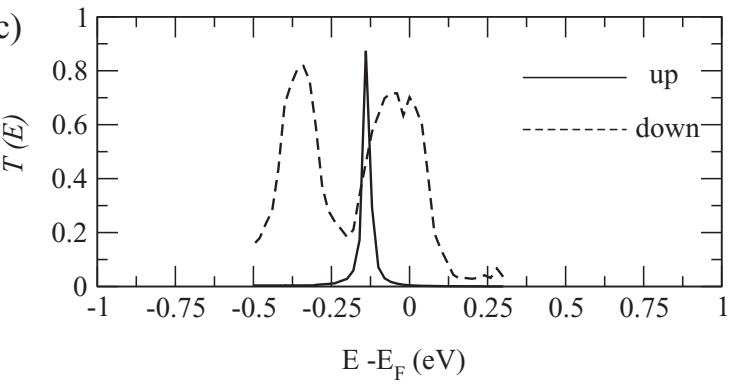

FIG. 2. Electronic and transport properties of the $\mathrm{Fe}(001)$ |quaterthiophene $\mid \mathrm{Fe}(001)$ nanojunction: (a) Spin-dependent PDOS on $d_{z x}$ orbitals of the Fe apex atom, (b) spin-resolved density of states on the quaterthiophene molecule, and (c) spin-resolved transmission function $T(E)$ as a function of the electron energy $E$ in the parallel magnetic configuration. Spin-up (spin-down) results are plotted by solid (dashed) lines. 
(a)

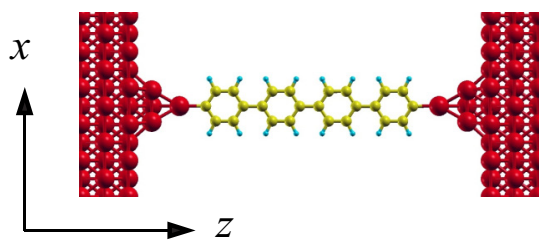

HOMO

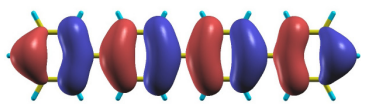

(b)

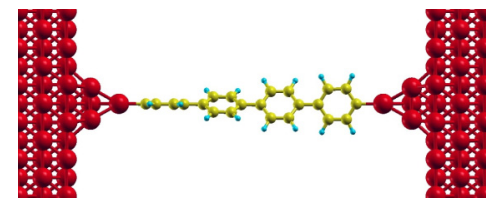

HOMO

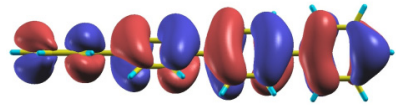

(c)

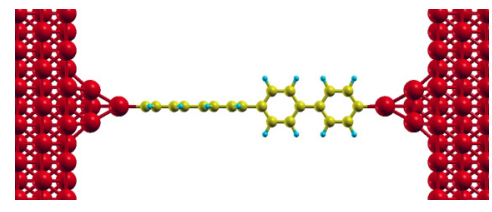

$\operatorname{HOMO}(\mathrm{x} 2)$

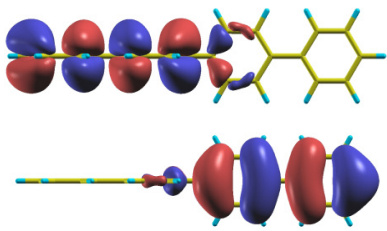

$$
G_{\downarrow} \approx 0.09 G_{0}, G_{\uparrow} \approx 0.00 G_{0} \quad G_{\downarrow} \approx 0.04 G_{0}, G_{\uparrow} \approx 0.00 G_{0} \quad G_{\downarrow} \approx 0.00 G_{0}, G_{\uparrow} \approx 0.00 G_{0}
$$

FIG. 3. Electronic and transport properties of the $p$-tetraphenyl molecule suspended between two Fe(110) electrodes. Three molecular conformations were considered: (a) a flat molecule, (b) a distorted molecule with $30^{\circ}$ rotation between adjacent phenyl cycles, and (c) a distorted molecule with $90^{\circ}$ rotation. Top panels present model geometries of molecular junctions. Middle panels show the charge-density isosurface plots for the HOMO of the free molecule. Note that the isosurfaces of positive and negative isovalues are shown in red and blue, respectively. Lower panels report the spin-resolved PDOS on the molecule, with the spin-up (spin-down) curves presented by solid (dashed) lines. At the bottom, the conductances for both spin channels are indicated.

of the free electrode [see Fig. 1(d), lower panel]. However, a slight splitting of the majority spin states near the Fermi level, as well as a small peak at about $-0.13 \mathrm{eV}$ for the majority spin states, arise due to the hybridization with the molecule. Clearly, the PDOS of the Fe apex atom is still dominated by the minority spin states near the Fermi level.

The transport properties of molecular junctions are determined by molecular orbitals located near the Fermi energy. In Fig. 2(b) we present the DOS projected on the molecule, where one can clearly see that the HOMO is very close to the Fermi energy, meaning that the HOMO is the main transport channel. We note that, unlike metallic electrodes, the molecular states are usually not described quite accurately within a standard DFT approach. A more accurate treatment using, for example, a $G W$ approximation is needed in many cases in order to improve the molecular level alignment (with respect to the Fermi level of the electrodes) and to correct for the HOMO-LUMO (lowest unoccupied molecular orbital) gap. It has been shown, however, that $G W$ corrections affect rather unoccupied orbitals (poorly described within the ground state DFT) while occupied levels (in particularly, the HOMO) are only slightly altered [23]. Since in our case the electron transport is controlled by the HOMO orbital, we hope that our mean-field DFT results are quite reliable. The spin-up resonance located at about $-0.13 \mathrm{eV}$ is very sharp due to the lack of appropriate symmetry states in the electrode for the majority spin near the Fermi energy. On the contrary, the HOMO state for the spin-down channel has a significantly broader structure (going from -0.5 to $0.13 \mathrm{eV}$, roughly) which reflects the increased hybridization with the electrode states. A similar effect (but less pronounced) has been also reported for the same molecular junction bridging two $\mathrm{Ni}(111)$ electrodes [17].

As a consequence, the remarkable difference in the conductance for the two spin channels is observed as shown in Fig. 2(c). For spin-down electrons, a significant and broad transmission peak is observed. It is located between -0.1 and $0.1 \mathrm{eV}$, with a maximum close to the Fermi energy. At the 
same time, the transmission coefficient in the spin-up channel is nearly zero at the Fermi level. For that reason, the transport is fully due to spin-down electrons-we find $G_{\downarrow} \approx 0.70 G_{0}$ and $G_{\uparrow} \approx 0.004 G_{0}$, for spin-down and spin-up polarizations, respectively.

$\mathrm{Fe}(110) \mid$ p-tetraphenyl $\mid \mathrm{Fe}(110)$. In order to understand the effect of a molecule on the spin-filtering efficiency, we now investigate another molecular junction made of $p$-tetraphenyl connecting two Fe(110) electrodes, as shown in Fig. 3. This molecule was chosen because it has a much smaller band gap of $0.4 \mathrm{eV}$ in the infinite chain configuration compared to the polythiophene chain of $1.4 \mathrm{eV}$. Therefore, we thought that the corresponding molecular junction will show a higher spin-down conductance.

We start by discussing the flat $p$-tetraphenyl junction as presented in Fig. 3(a). From the charge-density isosurface plots of the HOMO level, we can clearly see the out-ofplane character of the HOMO orbital. Compared to the quaterthiophene junction discussed above, the HOMO was found, however, to be shifted by about $-0.48 \mathrm{eV}$ with respect to the Fermi energy, which results in the lower spin-down conductance, $G_{\downarrow} \approx 0.09 G_{0}$. The conductance in the spin-up channel is again almost zero, indicating a $100 \%$ spin filtering.

We next investigate the distortion effect of the molecule on its transport property. Among the three configurations considered (see Fig. 3), we have found that the one with phenyl rings rotated consequently by $30^{\circ}$ around the $Z$ axis [Fig. 3(b)] is the most favorable, explicitly, 0.12 and $0.27 \mathrm{eV}$ lower in energy than the flat molecule [Fig. 3(a)] and the one with two parts rotated by $90^{\circ}$ [Fig. 3(c)], respectively. Interestingly, the HOMO level moves away from the Fermi energy when the degree of the distortion is increased, i.e., the HOMO moves to about -0.52 and $-0.75 \mathrm{eV}$ for distortions of $30^{\circ}$ and $90^{\circ}$, respectively. As a result, a much smaller conductance of $G_{\downarrow} \approx 0.04 G_{0}$ is found for $30^{\circ}$ distortion compared to the flat molecule. Interestingly, for the $90^{\circ}$ distortion, we have found almost zero conductance in both spin channels. This is attributed to two reasons: (i) The twofold degenerate HOMOs are strongly localized on either the right or left sides [Fig. 3(c)] and are thus completely decoupled from the corresponding electrode, which can be seen as the breaking of the conjugation; and (ii) the HOMO is positioned very far from the Fermi energy. In this case, the molecular junction can be seen as two uncoupled molecules sandwiched between the electrodes, which does not allow the electric current to pass.

Summary. We present ab initio quantum transport calculations of spin-polarized electron transport through a special class of $\pi$-conjugated molecules bridging two ferromagnetic electrodes. By analyzing systematically the PDOS on the apex atom of free ferromagnetic electrodes for different materials as well as for different crystallographic orientations, we selected the $\mathrm{Fe}(110)$ as an optimal electrode for efficient spin injection. Here, a perfect DOS of appropriate symmetry is obtained in the spin-down channel-large and smooth around the Fermi energy-while the spin-up contribution is negligibly small. A perfect spin filtering has been found for both quaterthiophene and $p$-tetraphenyl molecular junctions bridging $\mathrm{Fe}(110)$ electrodes, which confirms the generality of our recently proposed symmetry mechanism to obtain fully polarized currents in single molecule nanojunctions [17]. Interestingly, a rather high spin-down conductance, $G_{\downarrow} \approx$ $0.70 G_{0}$, is found for a quaterthiophene junction, while a much smaller value, $G_{\downarrow} \approx 0.09 G_{0}$, is found for a $p$-tetraphenyl one. Therefore, the quaterthiophene (and, more generally, polythiophene molecules) seems to be the best candidate for the spin-filtering property, which is directly related to a very close position of its HOMO to the Fermi energy, $E=-0.13 \mathrm{eV}$. In the case of $p$-tetraphenyl, the conductance was found to decrease when the phenyl rings are rotated with respect to the others, and gets completely cut in the orthogonal configuration. Finally, it should be emphasized that no antiparallel magnetic alignment of two electrodes was studied explicitly in this Rapid Communication, however, we can argue that the perfectly spin-polarized conductances reported here should directly result also in ideally infinite ratios of MR, as has been verified in our previous study [17]. We believe that our results will be important for future electronics and digital information technologies based on hybrid metal/organics components.

Acknowledgments. This work was performed using computation resources from GENCI-[TGCC] project (Grants No. 2015097416 and No. 2016097416).
[1] A. R. Rocha, V. M. Garcia-Suarez, S. W. Bailey, C. J. Lambert, J. Ferrer, and S. Sanvito, Nat. Mater. 4, 335 (2005).

[2] S. Sanvito, Nat. Phys. 6, 562 (2010).

[3] C. Barraud, P. Seneor, R. Mattana, S. Fusil, K. Bouzehouane, C. Deranlot, P. Graziosi, L. Hueso, I. Bergenti, V. Dediu et al., Nat. Phys. 6, 615 (2010).

[4] D. Sun, L. Yin, C. Sun, H. Guo, Z. Gai, X.-G. Zhang, T. Z. Ward, Z. Cheng, and J. Shen, Phys. Rev. Lett. 104, 236602 (2010).

[5] X. Fei, G. Wu, V. Lopez, G. Lu, H.-J. Gao, and L. Gao, J. Phys. Chem. C 119, 11975 (2015).

[6] D. Çakır, D. M. Otálvaro, and G. Brocks, Phys. Rev. B 89, 115407 (2014).

[7] K. Yoshida, I. Hamada, S. Sakata, A. Umeno, M. Tsukada, and K. Hirakawa, Nano Lett. 13, 481 (2013).

[8] S. Schmaus, A. Bagrets, Y. Nahas, T. K. Yamada, A. Bork, M. Bowen, E. Beaurepaire, F. Evers, and W. Wulfhekel, Nat. Nanotechnol. 6, 185 (2011).
[9] A. Bagrets, S. Schmaus, A. Jaafar, D. Kramczynski, T. K. Yamada, M. Alouani, W. Wulfhekel, and F. Evers, Nano Lett. 12, 5131 (2012).

[10] L. L. Tao, S. H. Liang, D. P. Liu, and X. F. Han, J. Appl. Phys. 114, 213906 (2013).

[11] S. L. Kawahara, J. Lagoute, V. Repain, C. Chacon, Y. Girard, S. Rousset, A. Smogunov, and C. Barreteau, Nano Lett. 12, 4558 (2012).

[12] N. Atodiresei, J. Brede, P. Lazić, V. Caciuc, G. Hoffmann, R. Wiesendanger, and S. Blügel, Phys. Rev. Lett. 105, 066601 (2010).

[13] D. Jacob, J. Fernández-Rossier, and J. J. Palacios, Phys. Rev. B 71, 220403 (2005).

[14] A. Smogunov, A. Dal Corso, and E. Tosatti, Phys. Rev. B 73, 075418 (2006).

[15] M. Häfner, J. K. Viljas, D. Frustaglia, F. Pauly, M. Dreher, P. Nielaba, and J. C. Cuevas, Phys. Rev. B 77, 104409 (2008). 
[16] R. Vardimon, M. Klionsky, and O. Tal, Nano Lett. 15, 3894 (2015).

[17] A. Smogunov and Y. J. Dappe, Nano Lett. 15, 3552 (2015).

[18] P. Giannozzi, S. Baroni, N. Bonini, M. Calandra, R. Car, C. Cavazzoni, D. Ceresoli, G. L. Chiarotti, M. Cococcioni, I. Dabo et al., J. Phys.: Condens. Matter 21, 395502 (2009).

[19] J. P. Perdew and A. Zunger, Phys. Rev. B 23, 5048 (1981).

[20] The plane wave basis was employed with an energy cutoff of 30 and 300 Ry for the wave functions and the charge density, respectively. A $(4 \times 4)$ in-plane periodicity was used in the $x y$ plane, perpendicular to the molecular junctions, in order to minimize unphysical molecule-molecule interactions. Four and three metallic layers were employed to model the left and right electrodes, respectively, and the periodicity in all three directions was assumed in electronic structure calculations. Ionic relaxations were carried out using a $(3 \times 3 \times 1) k$-point mesh while a more dense $(6 \times 6 \times 1)$ mesh was used for the density of states analysis.
[21] A. Smogunov, A. Dal Corso, and E. Tosatti, Phys. Rev. B 70, 045417 (2004).

[22] S. Y. Quek, J. B. Neaton, M. S. Hybertsen, E. Kaxiras, and S. G. Louie, Phys. Rev. Lett. 98, 066807 (2007).

[23] M. Strange, C. Rostgaard, H. Häkkinen, and K. S. Thygesen, Phys. Rev. B 83, 115108 (2011).

[24] G. Reecht, H. Bulou, F. Scheurer, V. Speisser, F. Mathevet, C. Gonzlez, Y. J. Dappe, and G. Schull, J. Phys. Chem. Lett. 6, 2987 (2015).

[25] L. Lafferentz, F. Ample, H. Yu, S. Hecht, C. Joachim, and L. Grill, Science 323, 1193 (2009).

[26] M. Kiguchi, O. Tal, S. Wohlthat, F. Pauly, M. Krieger, D. Djukic, J. C. Cuevas, and J. M. van Ruitenbeek, Phys. Rev. Lett. 101, 046801 (2008).

[27] T. Yelin, R. Korytár, N. Sukenik, R. Vardimon, B. Kumar, C. Nuckolls, F. Evers, and O. Tal, Nat. Mater. 15, 444 (2016). 